\title{
EDITORIAL
}

\section{HIGH TECHNOLOGY MEDICINE AND THE ELDERLY IN BRITAIN}

\author{
Bryan Jennett \\ University of Glasgow
}

Geriatrics as a specialty was born in Britain, the first professor being at my own University of Glasgow. Britain also pioneered the hospice movement that recognized explicitly the need to withdraw active treatment once the outlook is hopeless. The movement was initially focused on cancer but has become more widely applied. Another feature of the United Kingdom is that many technologies are less available there than in the United States: for instance, only a tenth as many intensive care beds per million population, a sixth as many coronary artery bypass graft (CABG) operations and computed tomography (CT) scanners, and a half to a third as many patients on dialysis. It might be expected therefore that overtreatment of the elderly by high technology would not be a major problem. But that is to underestimate the "imperative to therapy," the impulse to rescue even when the patient is old.

Indeed, the elderly figure disproportionately in the acute hospital beds in Britain as they do in the United States. Although over-65-year-olds make up only $14 \%$ of the United Kingdom population, they account for almost $50 \%$ of the patients in acute hospital beds, $40 \%$ in surgical wards, $30 \%$ in general intensive care units, and up to $20 \%$ of those having open heart surgery. In coronary care units, some $60 \%$ are over 60 years and $20 \%$ over 70 years. These figures may not seem very surprising because the old more often become ill. But because critical illness in the elderly is often terminal illness, the question is whether such treatment is sometimes inappropriate, that is, unsuccessful, unkind, or unwise (2).

There is also concern in the United Kingdom that the inadequate provision of chronic care for the elderly may stem partly from too much of the NHS budget being used in the acute hospital sector for high technology medicine. This has led to health departments recommending the allocation of resources in favor of chronic care-a policy that ignores the appropriate need of many elderly patients for high technology medicine. Although supposedly in the interests of the elderly, such a policy could prove counter-productive. If therapeutic technologies were to become even more strictly rationed, it is likely that some older patients who could benefit from such treatment might be denied access.

When demand for technology exceeds supply, advanced age is a readily available and widely accepted means of selection. However, geriatricians often em- 
phasize that the results of treatment in the elderly compare well with younger patients. But these good results almost certainly reflect the more stringent selection criteria applied to the elderly. Several biological features of aging adversely affect outcome. These include more frequent multiple pathology, more frequent complications, and a reduced capacity to withstand physiological perturbations because the reserve of all organs decreases linearly from the age of 30 years. When care is taken to standardize for severity of illness before relating age to outcome, the significant effect of increasing age on prognosis becomes clear. The international data bank of severe head injuries in Glasgow (which includes cases from California) shows that mortality increases by $3.6 \%$ for each year over 3.5 years (6).

Apart from these biological influences on outcome, there are personal and social aspects of advancing age. Older people are less likely to consent to heroic measures when they are given the opportunity to express a preference. Provided that patients are competent, they should be required to give consent not only for surgery but for other rescue procedures such as intensive care and dialysis. Consent should be sought not only for the initiation of intensive measures but also for the continuation of such support when it has become clear that prolonged, good quality survival is no longer possible. Securing such consent requires that information be given about the probability of success and about what disabilities are expected in the short and the long term.

There is good evidence that older patients are more willing to take risks of immediate mortality from interventions, perhaps because the inevitability of death is more obvious to them. But they are also more tolerant of continuing disability if this is mainly a limitation in activities rather than persistence of distressing symptoms. However, even if a successful immediate outcome is achieved, life expectancy is obviously more limited in older patients, so estimated overall benefit (e.g., quality-adjusted life years) is bound to be less than in younger patients. Indeed, elderly patients often put a lower value on their own survival than on that of younger people. Two European countries recently suggested that rescue procedures should not be undertaken for patients over 65 years unless a five year expectancy of life was probable. Yet such procedures are commonly undertaken in younger patients when the outlook is far less favorable than the five year life expectancy, in particular, when treating cancer by major surgery.

In Britain there is no such general moratorium on the treatment of elderly patients, although much has been made of their limited access to dialysis. Indeed, formal and explicit rules for restricting therapy are rare. There still appears to be no British intensive care unit that has explicit guidelines even for withholding resuscitation although these have been commonplace in the United States for years. However, a report on cardiopulmonary resuscitation in an English geriatric ward did emphasize that this was not used for patients with irreversible coma, severe dementia, total dependency due to chronic illness or with terminal malignancy (1). Moreover, some British doctors have proposed that under some circumstances even the maintenance of fluid and nutritional intake could be regarded as extraordinary care for some hopelessly ill patients whatever their age (5). Most British doctors are still as prone as colleagues elsewhere to what I have called the cycle of commitment-once treatment is started there is reluctance to abandon it even when it has become plain that it can do no good (3). 
Age should be given its appropriate place in decisions about the initiation and continuation of rescue therapy. Whether treatment is appropriate depends on the chance of restoring life of quality for long enough to be worth the burden of treatment to the patient, to the family, and to society. It also depends on a reasonable certainty that intervention will not do more harm than good-a value judgment about the balance of burdens and benefits (4). It is also necessary to be reasonably sure that the course followed is what the patient wants or would have wanted were he/she able to tell us. We should also be satisfied that we are not either denying treatment or insisting on it for the benefit of the doctors rather than the patient. We should not exclude an elderly patient from access to a procedure so as to preserve the statistics of success in a series to be reported, because this may deprive a patient of a reasonable chance of relief. Nor should we treat an elderly patient in order to save the trouble of defending ourselves against charges of inadequate care when treatment would be against the patient's wishes or best interests.

The best safeguard against inappropriate treatment is for institutions and individual services to evolve written guidelines about good practice in the field of rescue therapy at all ages. Such a prior consensus should be based on the best available data about prognosis-with and without certain interventions. It is dishonest to hide behind a cloak of supposed ignorance, to maintain with mock humility that we do not know who will and who will not respond to therapy. For many situations good data are available about expected outcome. Where they are not we should set about collecting the data that are necessary to improve decision making. Decisions should be based on data-not on slogans, on impulses, or on cowardice.

\section{REFERENCES}

1. Gulati, R. S., Bhan, G. L., \& Horan, M. A. Cardiopulmonary resuscitation of old people. Lancet, 1983, 2, 267-9.

2. Jennett, B. Inappropriate use of intensive care. British Medical Journal, 1984, 289, 1709-11.

3. Jennett, B. Intensive Care for the Elderly. International Journal of Technology Assessment in Health Care, 1985, 1, 7-19.

4. Jennett. B. High techmology medicine-Benefits and burdens 12 nd ed.). Oxford and New York: Oxford University Press. 1986.

5. Jennett. B. Decisions to limit treatment. Lance'. 1987.

6. Teasdale. G.. Skene, A.. Spiegelhalter. D.. \& Murray. L. Age. severity, and outcome of head injury. In Grossman. R. J.. \& Gildenberg. P. L. (eds.). Head injury: Basic and clinical aspects. New York: Raven Press. 1982. 